\title{
Effect of pneumoperitoneum on the recovery from intense neuromuscular blockade by rocuronium in healthy patients undergoing laparoscopic surgery
}

\author{
Hong Soon Kim, Dong Chul Lee, Mi Geum Lee, Woon Rak Son, and Yong Beom Kim \\ Department of Anesthesiology and Pain Medicine, Gachon University of Medicine and Science, Incheon, Korea
}

Background: This study investigated the effect of pneumoperitoneum on the recovery from intense neuromuscular blockade by rocuronium in healthy patients undergoing laparoscopic abdominal surgery.

Methods: Thirty adult patients undergoing laparoscopic abdominal surgery were studied. Anesthesia was induced with $1.5 \mathrm{mg} / \mathrm{kg}$ of propofol, $12 \mathrm{ug} / \mathrm{kg}$ of alfentanil and $0.6 \mathrm{mg} / \mathrm{kg}$ of rocuronium and maintained with $2 \mathrm{vol} \%$ of sevoflurane and $0.05-0.2 \mu \mathrm{g} / \mathrm{kg} / \mathrm{min}$ remifentanil. The neuromuscular relaxation was monitored by Train-of-Four (TOF) and posttetanic count (PTC). Additional rocuronium of $0.2 \mathrm{mg} / \mathrm{kg}$ was administered for deep neuromuscular blockade at $30 \mathrm{~min}$ after pneumoperitoneum. Before (PPpre) and $30 \mathrm{~min}$ after pneumoperitoneum (PPpost), PTC was measured at $6 \mathrm{~min}$ intervals. The relationship between PTC and the time interval to reappearance of T1 response was observed.

Results: The mean \pm SD of the intervals between the detection of 4 counts of the PTC and the first response to TOF stimulation was $13.0 \pm 1.1 \mathrm{~min}$ and $16.4 \pm 6.3 \mathrm{~min}$ PPpre and PPpost, respectively $(\mathrm{P}=0.20)$. There were significant negative relationships between PTC observed and the time interval to reappearance of T1 response (adjusted $\mathrm{R}^{2}=0.869, \mathrm{P}<0.001$ for PPpre data, and adjusted $\mathrm{R}^{2}=0.561, \mathrm{P}<0.001$ for PPpost data). Comparing the difference of regression equation between PPpre and PPpost data using a parallelism test, there was no statistically significant difference $(\mathrm{P}=0.193)$.

Conclusions: This study showed that PP with intra-abdominal pressure at the level of 13-14 mmHg did not affect the recovery from intense neuromuscular blockade by rocuronium in healthy patients undergoing laparoscopic abdominal surgery. (Korean J Anesthesiol 2014; 67: 20-25)

Key Words: Neuromuscular blockade, Neuromuscular monitoring, Pneumoperitoneum, Rocuronium.

Received: October 23, 2013. Revised: November 25, 2013. Accepted: December 9, 2013.

Corresponding author: Yong Beom Kim, M.D., Department of Anesthesiology and Pain Medicine, Gachon University of Medicine and Science, 21, Namdong-daero 774beon-gil, Namdong-gu, Incheon 405-760, Korea. Tel: 82-32-460-3400, Fax: 82-32-469-6319, E-mail: fred0314@gilhospital.com (c) This is an open-access article distributed under the terms of the Creative Commons Attribution Non-Commercial License (http:// creativecommons.org/licenses/by-nc/3.0/), which permits unrestricted non-commercial use, distribution, and reproduction in any medium, provided the original work is properly cited. 


\section{Introduction}

Laparoscopic procedures have replaced a variety of open intra-abdominal surgeries, because it is a relatively non-invasive procedure with fast recovery and less pain [1]. However, laparoscopy requires a formation of the working area within the peritoneal cavity, and this is commonly achieved by carbon dioxide $\left(\mathrm{CO}_{2}\right)$ pneumoperitoneum (PP), which raises concerns regarding the physiologic adverse effects [2,3]. An increased intra-abdominal pressure by $\mathrm{CO}_{2}$ inflation may cause significant changes in the hepatic function and blood flow [4,5].

Neuromuscular block is necessary for optimizing surgical field during pneumoperitoneum [6] and preventing abdominal compartment syndrome [7]. Post tetanic count (PTC) is a reliable method for evaluating the recovery from an intense neuromuscular blockade, because post tetanic twitch response appears earlier than that of the train-of-four (TOF) response during recovery from a neuromuscular blockade [8]. A close correlation has been shown between PTC and TOF recovery from an intense neuromuscular blockade by rocuonium [9]. Rocuronium bromide is a widely used non-depolarizing neuromuscular blocking agent, because of its rapid onset and intermediate duration of action. Although its elimination pathway remains unclear, some hepatic elimination of compound in humans might be expected. In patients with decreased hepatic function, rocuronium pharmacodynamic and pharmacokinetic alterations have been reported [10-12]. In addition, Wang et al. [13] reported that the effect of rocurnium was prolonged in patients with obstructive jaundice, and cautioned that monitoring postoperative residual neuromuscular blockade should be needed in these patients. To date, no report has been issued regarding the effects of PP on the relationship between PTC and recovery from an in- tense neuromuscular blockade. Thus, this study investigated the effect of pneumoperitoneum on this relationship in patients undergoing laparoscopic abdominal surgery. We hypothesized that a transient decrease in the hepatic and renal functions induced by pressure effect of pneumoperitoneum might result in delayed recovery from intense neuromuscular blockade by rocuronium.

\section{Materials and Methods}

After an approval from the Institutional Review Board, we obtained written informed consents from all patients. Thirty patients, aged 18-65 years with American Society of Anesthesiologists physical status I or II and undergoing laparoscopic abdominal surgery, were studied prospectively. Patients with known neuromuscular disorder, hepatic and renal diseases, past history of using drugs that might alter neuromuscular transmission or metabolism of rocuronium, and/or body mass index of $<18 \mathrm{~kg} / \mathrm{m}^{2}$ or $\geq 25 \mathrm{~kg} / \mathrm{m}^{2}$, were excluded. Patients in whom TOF measurements were made when peripheral body temperature was below $32^{\circ} \mathrm{C}$ were excluded from the study. The study protocol was summarized in Fig. 1. One hour before surgery, 2-3 mg of midazolam and $0.2 \mathrm{mg}$ of glycopyrrolate were administered intramuscularly as premedication. On arrival to the operating room, patients were monitored with standard anesthetic monitors, including electrocardiogram, pulse oxymeter, and non-invasive arterial pressure. Conventional inhalation anesthesia with 2 vol\% of maintaining end-tidal concentration of sevoflurane was performed with inducing dose of; $1.5 \mathrm{mg} / \mathrm{kg}$ of propofol, $12 \mu \mathrm{g} / \mathrm{kg}$ of alfentanil, and $0.6 \mathrm{mg} / \mathrm{kg}$ of rocuronium. Adjuvant analgesics during surgery were made with $0.05-0.20$ $\mu \mathrm{g} / \mathrm{kg} / \mathrm{min}$ remifentanil intravenously. After tracheal intubation, the lungs were ventilated with a tidal volume of $7-10 \mathrm{ml} / \mathrm{kg}$ and

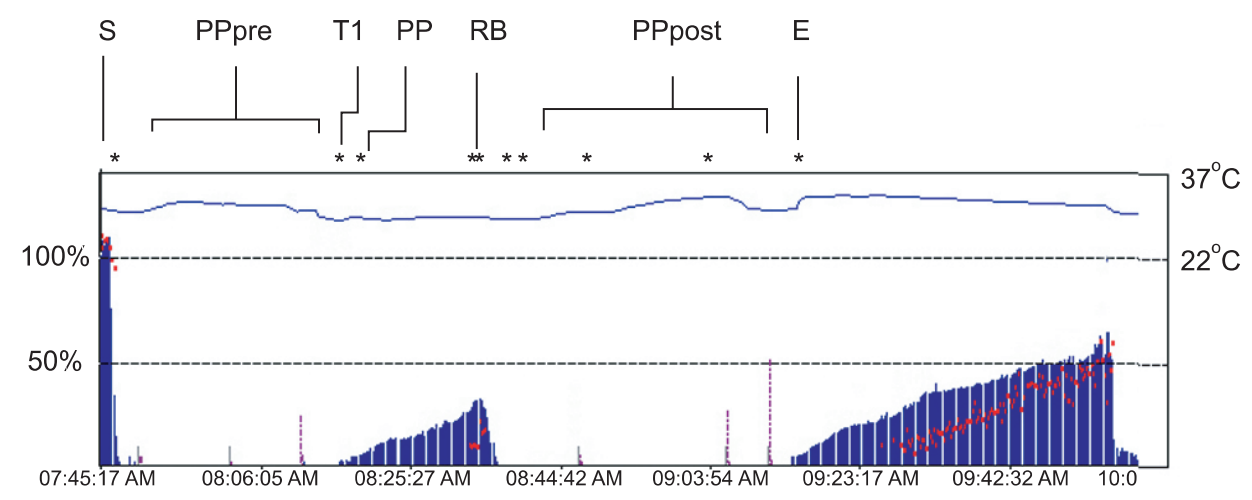

Fig. 1. Schematic sample of time course in this study. Trace recording of stabilization period before anesthesia induction and that of data obtained after end of study are truncated. S: the time at which intubating dose of rocuronium is administered. PPpre: the time during obtaining data before pneumoperitoneum. T1: indicates the time at which the first response of train of four (TOF) stimulation is reappeared. RB: the point at which the boost dose of rocuronium is administered. PP: indicates the time of initiation of pneumoperitoneum. PPpost: indicates the time during obtaining data for PPpost (data obtained from 30 minutes after pneumoperitoneum). E: indicates the point of study termination (point at which reappearance of the first response of TOF again). Study time course was scheduled to finish before deflation of peritoneal cavity had made in the middle of surgery. 
respiratory rate of $8-12$ breaths/min to maintain an end-tidal carbon dioxide concentration $\left(\mathrm{ETCO}_{2}\right)$ of $30-35 \mathrm{mmHg}$ at a $60 \%$ inspired oxygen with air. The tidal volume and respiratory rate was reset to maintain an $\mathrm{ETCO}_{2}$ between $35-40 \mathrm{mmHg}$ after pneumoperitoneum. The intraabdominal pressure was kept at a level of 13-14 mmHg. When a TOF ratio was more than 0.7 after the end of the operation, a reversal of neuromuscular blockade was achieved. After the patient was brought to the post anesthetic care unit (PACU), a residual neuromuscular blockade was monitored for about 1 hour with random time interval. To prevent the patient's inconvenience, we usually checked the clinical signs, such as hand grip for 5 second or leg raising for 5 second as the residual neuromuscular blockade monitoring. If these result was uncertain or ambiguous, objective neuromuscular monitor (TOF-watch SX ${ }^{\circledR}$, Organon Ltd, Dublin, Ireland) was applied and check TOF ratio with uncalibrated manner repeatedly until this ratio was shown more than 0.8 . When the patient was able to accomplish all of these test and other criteria for discharge from PACU was met, the patient was brought to the casual ward.

The neuromuscular relaxation was monitored with a TOFwatch $\mathrm{SX}^{\circledR}$ (Organon Ltd, Dublin, Ireland) through a transducer, which is attached to the volar surface of the thumb. For preload tension of the thumb, hand adapter for acceleration transducer (Organon Ltd, Dublin, Ireland) was applied throughout the investigation. The stimulation electrodes were placed over the ulnar nerve to give continuous supramaximal stimulation. The distance between electrodes was about $5 \mathrm{~cm}$. The skin temperature was measured at thenar eminence using the temperature sensor of TOF-watch $\mathrm{SX}^{\circledR}$. After losing consciousness, standard zero-calibration of neuromuscular monitoring was achieved (built-in calibration mode, CAL2, in the TOFwatch $S X^{\circledR}$ ). When all twitch responses of the TOF stimulation ( $2 \mathrm{~Hz}$ every $15 \mathrm{sec}, 200 \mu \mathrm{sec}$ duration) was faded and vanished after rocuronium injection, before pneumoperitoneum (PPpre), PTC was serially measured at 5 minute intervals. These cycles were repeated until the first reaction (T1) to TOF stimulation was detected (Fig. 1). At 30 minutes after pneumoperitoneum (PPpost), additional rocuronium of $0.2 \mathrm{mg} / \mathrm{kg}$ was administered for a deep neuromuscular blockade, followed by performing the same measurement and collected data for PPpost. During these cycles, the TOF mode was reinstituted with 15-second interval.

The sample size was calculated based on a previous study [14]. To detect the $20 \%$ mean difference between PPpre and PPpost in the interval between appearance of PTC and first response of TOF stimulation during sevoflurane anesthesia at a significant level of $5 \%$ and a probability power of $80 \%$; this study required at least 26 patients per group. The sample size was increased to 30 patients per group, assuming a possible dropout rate of $15 \%$. SPSS 13.0 (SPSS Inc., Chicago, IL, USA) was used for statistical analyses. Data are expressed as the mean \pm SD (ranges) or number of patients. The distribution of all measure and calculated data are tested by Kolmogorov-Smirnov tests. Student's t-test and regression were used to compare data. Significance was defined as $\mathrm{P}<0.05$. The relationship between post-tetanic count (PTC) and the time to the first response to TOF before (PPpre) and 30 min after pneumoperitoneum (PPpost) was plotted with exponential fitted curves.

\section{Results}

Thirty patients were initially enrolled, but one of these patients was excluded from the analysis due to subcutaneous emphysema with severe hypercarbia during pneumoperitoneum. Patient characteristics and peri-operative data are listed in Table 1. There were no significant changes in aspartate transaminase (AST), alanine transaminase (ALT), total bilirubin and $\gamma$-glutamyl transferase $(\gamma$-GT) after the surgery compared with preoperative data.

The mean \pm SD of the interval between the detection of four counts of PTC and the first response to TOF stimulation was $13.0 \pm 1.1 \mathrm{~min}$ and $16.4 \pm 6.3 \mathrm{~min}$ PPpre and PPpost, respectively $(\mathrm{P}=0.20)$. The number of PTC (independent variables) observed at a given time and the time from then to return of TOF stimulation (dependent variable) were tested for goodness of fit to linear and nonlinear models. The best fits to the data were obtained using the exponential model in PPpre and PPpost data. There were significant negative relationships between PTC observed and the time interval to reappearance of T1 response

Table 1. Patient Characteristics and Amount of Rocuronium Used

\begin{tabular}{|c|c|c|}
\hline Variables & \multicolumn{2}{|c|}{ Data } \\
\hline Gender (M/F) & \multicolumn{2}{|c|}{$16 / 13$} \\
\hline Age (yr) & \multicolumn{2}{|c|}{$48.6 \pm 8.5$} \\
\hline BMI $\left(\mathrm{kg} / \mathrm{m}^{2}\right)$ & \multicolumn{2}{|c|}{$23.7 \pm 3.0$} \\
\hline ASA I/II & \multicolumn{2}{|c|}{$18 / 11$} \\
\hline Penumoperitoneum time ( $\mathrm{min}$ ) & \multicolumn{2}{|c|}{$75[40-130]$} \\
\hline \multicolumn{3}{|l|}{ Type of laparoscopic surgery } \\
\hline Distal gastrectomy & \multicolumn{2}{|c|}{13} \\
\hline Vaginal hysterectomy & \multicolumn{2}{|c|}{8} \\
\hline Lower anterior resection & \multicolumn{2}{|c|}{8} \\
\hline Serum enzyme level & Preoperative & Postoperative \\
\hline AST (U/L) & $22.6 \pm 6.6$ & $27.8 \pm 10.8$ \\
\hline $\operatorname{ALT}(\mathrm{U} / \mathrm{L})$ & $22.7 \pm 10.2$ & $25.8 \pm 15.4$ \\
\hline T-bil (mg/dl) & $0.7 \pm 0.3$ & $0.9 \pm 0.4$ \\
\hline$\gamma-\mathrm{GT}(\mathrm{U} / \mathrm{L})$ & $25.4 \pm 16.3$ & $26.9 \pm 14.3$ \\
\hline
\end{tabular}

Data are expressed as mean \pm SD or median [range] or number of patients. BMI: body mass index, ASA: American Society of Anesthesiologists, AST: aspartate transaminase, ALT: alanine transaminase, T-bil: total bilirubin, $\gamma$-GT: gamma glutamyl transferase (Normal upper limits of AST, ALT, T-bil and $\gamma$-GT in our institute labs are $40 \mathrm{U} / \mathrm{L}, 40 \mathrm{U} / \mathrm{L}, 1.2$ $\mathrm{mg} / \mathrm{dl}$ and $45 \mathrm{U} / \mathrm{L}$ respectively). There are no significant differences in serum enzyme levels between preoperative and postoperative data. 


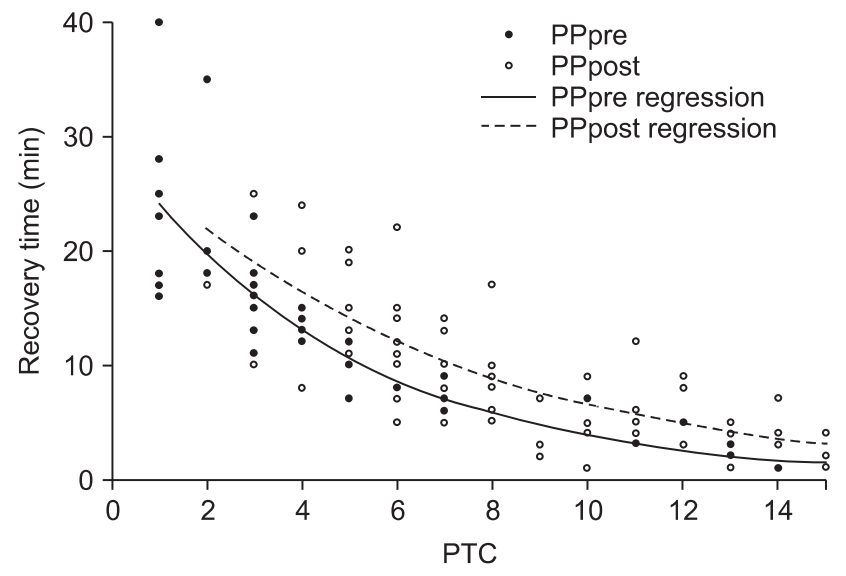

Fig. 2. The relationship between post-tetanic count (PTC) and the time to the first response to TOF before (PPpre) and $30 \mathrm{~min}$ after pneumoperitoneum (PPpost). Exponential fitted curves are shown. Assuming that the regression equation is $\mathrm{y}=\mathrm{ae}^{-\mathrm{bx}}$, there is a strong correlation with PTC and T1 reappearance time interval before pneumoperitoneum (adjusted $\mathrm{R}^{2}=0.869, \mathrm{P}<0.001$ ) and a weak correlation after pneumoperitoneum (adjusted $\mathrm{R}^{2}=0.561, \mathrm{P}<0.001$ ). In a comparison of the differences of regression equation between before and after pneumoperitoneum using parallelism test, there is no significant difference $(\mathrm{P}=0.193)$. PPpre: the time during obtaining data before pneumoperitoneum. PPpost: indicates the time during obtaining data for data obtained from 30 minutes after pneumoperitoneum.

(adjusted $\mathrm{R}^{2}=0.869, \mathrm{P}<0.001$ for PPpre data, and adjusted $\mathrm{R}^{2}=$ $0.561, \mathrm{P}<0.001$ for PPpost data) (Fig. 2). In comparing a difference of the regression equation between PPpre and PPpost data using parallelism test, there was no statistically significant difference $(\mathrm{P}=0.193)$.

\section{Discussion}

This study showed that pneumoperitoneum with intraabdominal pressure at the level of 13-14 $\mathrm{mmHg}$ did not affect the overall recovery from an intense neuromuscular blockade by rocuronium in healthy patients undergoing laparoscopic abdominal surgery.

In previous animal studies, it was observed that rocuronium may have been eliminated mainly via the liver. Three-fourth of intravenous rocuronium was found in the bile and liver homogenate [15]. Magorian et al. [16] found that hepatic dysfunction increased the volume of distribution of rocuronium, but did not alter its clearance. A previous study of Schlachtrupp et al. [17] reported that $15 \mathrm{mmHg}$ of pneumoperitoneum during $24 \mathrm{~h} \mathrm{im-}$ paired the function and morphological changes in the liver and elevated the serum ALT and alkaline phosphatase levels with low grade liver necrosis. In the present study, since the median pneumoperitoneum time was less than 75 min (range 40-130 $\mathrm{min}$ ), we did not observe significant increments of hepatic profiles and changes in the recovery from an intense neuromuscular blockade. This result is consistent with a previous report which represented a decrease in liver microvascular perfusion during $6 \mathrm{~h}$ of pneumoperitoneum, but showed compensatory improvement of liver blood flow after desufflation; and they concluded that prolonged pneumoperitoneum does not hamper the liver function [18]. The range of pneumoperitoneum time was wide from 40 minutes to 130 minutes. But during laparoscopic assisted subtotal gastrectomy or anorectal surgery, pneumoperitoneum was often released for manual manipulation or procedure outside the peritoneal cavity. In this regard, we tried to end this experiment before release of pneumoperitoneum. That's why we planned to obtained PPpost at the 30 minutes after initiation of pneumoperitoneum.

In this study, the average interval between the detection of four counts of PTC and the reappearance of T1 before pneumoperitoneum was 13.0 minutes. Conventionally, deep neuromuscular block state for sufficient blockade of diaphragmatic movement is considered as the point of PTC of less than 5 counts which is checked at the adductor policis muscle [19]. We decided to use the point at the detection of 4 PTC as a reference point. As a result, there was no significant difference in this time between the before and after pneumoperitoneum . Additionally, we observed a strong correlation with PTC and T1 reappearance time interval before pneumoperitoneum (adjusted $\mathrm{R}^{2}=0.869$ ) and a weak correlation after PP (adjusted $\mathrm{R}^{2}=0.561$ ). Considering the fact that there was no significance in the recovery from an intense neuromuscular block between continuous infusion and single bolus administration of rocuronium [20], a possible explanation for the decrement in correlation coefficient between before and after PP is the effects of variable intra-abdominal pressure and position. A previous animal study reported that changes in the intra-peritoneal pressure and position significantly influence the hepatic blood flow [21]. They demonstrated that both, the portal and hepatic arterial blood flow were reduced in relation to an increased intra-abdominal pressure and head-up position rather than the systemic hemodynamic parameters [21]. But this reduction of blood flow often occurred when the intraabdominal pressure is more than $20 \mathrm{mmHg}$. We tried to limit the intra-abdominal pressure at a level of 13-14 $\mathrm{mmHg}$, and considering the fact that there was no significant change in serum enzyme level which is related to the liver function (Table 1), it may reasonable to explain that our results may not be caused by the irreversible decrease in liver function. During surgical manipulation, set point of intraabdominal pressure could not be fixed. Transient elevation of intraabdominal pressure up to the upper limit alarm set point. Also, laparoscopic low anterior resection and gynecologic surgery both require the involvement of Trendelenburg position, while laparoscopic stomach surgery needs the reverse Trendelenburg position. Considering all of these facts, we thought that our results may be by the mechani- 
cal effect of pneumoperitoneum on intraabdominal organ and not by the irreversible impairment of liver function. Nowadays, intraabdominal pressure for induced pneumoperitoneum often is set as low as $7-8 \mathrm{mmHg}$ because it guarantees advantage in postoperative complication such as overall postoperative pain, preservation of pulmonary function, or at least there are no differences postoperative outcome [22,23]. But this low pressure method has some drawbacks. One of these is the lack of surgeon's experience [23]. Though there were no differences in the intraoperative or postoperative complication, the surgeon often experiences difficulty in obtaining optimal sight or dissection space and these drawbacks is more severe when using suction. Inexperienced surgeon asks to increase intraabdominal pressure up to the conventional setting of more than $12 \mathrm{mmHg}$.

The present study has several limitations that warrant consideration. First, we only tested representative hepatic profile, such as AST, ALT, $\gamma$-GT and total bilirubin. Although these laboratory findings could reflect the hepatic parenchymal changes or obstruction, they could not indicate the hepatic clearance or metabolism of certain drugs, and could not measure the direct changes of hepatic blood flow. Second, sevoflurane significantly potentiate the effect of neuromuscular blocking agent and its monitoring [24]. Because the effect of pneumoperitoneum on respiratory function, we could not completely excluded the in- fluence of inhalation agent even though we fixed the end-tidal concentration of sevoflurane. But sevoflurane is least potent on the effect of neuromuscular blockade and there was no effect of anesthetic agents on recovery profile [25]. And this effect of inhalation agent on muscle takes more than 30 minute to affect the muscle strength [26]. So we choose this conventional inhalation agent for our study. To be free from this argument, further study with total intravenous anesthesia might be needed. Third, we excluded the operations that required hepato-biliary manipulation because we wanted to eliminate the possibility of other mechanical factors except the pure pneumoperitoneum effects. Jeong et al. [27] reported that hepatic manipulation during surgical procedure is the main cause of changes in the hepatic function rather than $\mathrm{CO}_{2}$ pneumoperitoneum. Thus, our results could apply to limited laparoscopic procedures.

In conclusion, we conclude that pneumoperitoneum with intra-abdominal pressure at the level of 13-14 mmHg did not affect the overall recovery from an intense neuromuscular blockade by rocuronium in healthy patients undergoing laparoscopic abdominal surgery. However, because individual recovery pattern at pneumoperitoneum is relatively inconsistent comparing with that of the normal (PPpre) state, we also conclude that the close neuromuscular monitoring should especially be mandatory during pneumoperitoneum.

\section{References}

1. Cuschieri A. Laparoscopic cholecystectomy. J R Coll Surg Edinb 1999; 44: 187-92.

2. Koivusalo AM, Lindgren L. Effects of carbon dioxide pneumoperitoneum for laparoscopic cholecystectomy. Acta Anaesthesiol Scand 2000; 44: 834-41.

3. Clarke RS, Doggart JR, Lavery T. Changes in liver function after different types of surgery. Br J Anaesth 1976; 48: 119-28.

4. Mogilner J, Sukhotnik I, Brod V, Hayari L, Coran AG, Shiloni E, et al. Effect of elevated intra-abdominal pressure in portal vein and superior mesenteric artery blood flow in a rat. J Laparoendosc Adv Surg Tech A 2009; 19 Suppl 1: S59-62.

5. Omari A, Bani-Hani KE. Effect of carbon dioxide pneumoperitoneum on liver function following laparoscopic cholecystectomy. J Laparoendosc Adv Surg Tech A 2007; 17: 419-24.

6. Staehr-Rye AK, Rasmussen LS, Rosenberg J, Juul P, Gätke MR. Optimized surgical space during low-pressure laparoscopy with deep neuromuscular blockade. Dan Med J 2013; 60: A4579.

7. Chiles KT, Feeney CM. Abdominal compartment syndrome successfully treated with neuromuscular blockade. Indian J Anaesth 2011; 55: 384-7.

8. Viby-Mogensen J, Howardy-Hansen P, Chraemmer-Jørgensen B, Ording H, Engbaek J, Nielsen A. Posttetanic count (PTC): a new method of evaluating an intense nondepolarizing neuromuscular blockade. Anesthesiology 1981; 55: 458-61.

9. Schultz P, Ibsen M, Østergaard D, Skovgaard LT. Onset and duration of action of rocuronium--from tracheal intubation, through intense block to complete recovery. Acta Anaesthesiol Scand 2001; 45: 612-7.

10. Kim SY, Hwang KH, Han JW. Clinical use of nondepolarizing neuromuscular relaxants; neuromuscular pharmacology in clinical anesthesia. 2nd ed. Edited by Kim SY: Seoul, Yomungak. 2011, pp 149-54.

11. van Miert MM, Eastwood NB, Boyd AH, Parker CJ, Hunter JM. The pharmacokinetics and pharmacodynamics of rocuronium in patients with hepatic cirrhosis. Br J Clin Pharmacol 1997; 44: 139-44.

12. Servin FS, Lavaut E, Kleef U, Desmonts JM. Repeated doses of rocuronium bromide administered to cirrhotic and control patients receiving isoflurane: A clinical and pharmacokinetic study. Anesthesiology 1996; 84: 1092-100.

13. Wang Z, Bao Y, Lu Z, Yu W. Is neuromuscular relaxation of rocuronium prolonged in patients with obstructive jaundice? Med Hypotheses 2011; 76: 100-1. 
14. Baykara N, Solak M, Toker K. Predicting recovery from deep neuromuscular block by rocuronium in the elderly. J Clin Anesth 2003; 15: 328-33.

15. Khuenl-Brady K, Castagnoli KP, Canfell PC, Caldwell JE, Agoston S, Miller RD. The neuromuscular blocking effects and pharmacokinetics of ORG 9426 and ORG 9616 in the cat. Anesthesiology 1990; 72: 669-74.

16. Magorian T, Wood P, Caldwell J, Fisher D, Segredo V, Szenohradszky J, et al. The pharmacokinetics and neuromuscular effects of rocuronium bromide in patients with liver disease. Anesth Analg 1995; 80: 754-9.

17. Schachtrupp A, Toens Ch, Hoer J, Klosterhalfen B, Lawong AG, Schumpelick V. A 24-h pneumoperitoneum leads to multiple organ impairment in a porcine model. J Surg Res 2002; 106: 37-45.

18. Hoekstra LT, Ruys AT, Milstein DM, van Samkar G, van Berge Henegouwen MI, Heger M, et al. Effects of prolonged pneumoperitoneum on hepatic perfusion during laparoscopy. Ann Surg 2013; 257: 302-7.

19. Ueda N, Muteki T, Tsuda H. What anesthesiologist should know about neuromuscular monitoring today? J Anesth 1992; 6: $192-206$.

20. Kim HS, Lee KC, Lee DC, Jang J, Kim YB. Comparison of recovery times from deep neuromuscular blockade between single bolus and continuous infusion of rocuronium. Anesth Pain Med 2009; 4: 336-40.

21. Junghans T, Böhm B, Gründel K, Schwenk W, Müller JM. Does pneumoperitoneum with different gases, body positions, and intraperitoneal pressures influence renal and hepatic blood flow? Surgery 1997; 121: 206-11.

22. Warlé MC, Berkers AW, Langenhuijsen JF, van der Jagt MF, Dooper PM, Kloke HJ, et al. Low-pressure pneumoperitoneum during plaparoscopic donor nephrectomy to optimize live doners' comfort. Clin Transplant 2013: 27; E478-83.

23. Joshipura VP, Haribhakti SP, Patel NR, Naik RP, Soni HN, Patel B, et al. A prospective randomized, controlled study comparing low pressure versus high pressure pneumoperitoneum during laparoscopic cholecystectomy. Surg Laparosc Endosc Percutan Tech 2009: 19; 234-40.

24. Suzuki T, Munakata K, Watanabe N, Katsumata N, Saeki S, Ogawa S. Augmentation of vecuronium-induced neuromuscular block during sevoflurane anaesthesia: comparison with balanced anaesthesia using propofol or midazolam. Br J Anaesth 1999; 83: 485-7.

25. Bock M, Klippel K, Nitsche B, Bach A, Martin E, Motsch J. Rocuronium potency and recovery characteristics during steady-state desflurane, sevoflurane, isoflurane or propofol anaesthesia. Br J Anaesth 2000; 84: 43-7.

26. Meretoja OA, Wirtavuori K, Taivainen T, Olkkola KT. Time course of potentiation of mivacurium by halothane and isoflurane in children. Br J Anaesth 1996; 76: 235-8.

27. Jeong GA, Cho GS, Shin EJ, Lee MS, Kim HC, Song OP. Liver function alterations after laparoscopy-assisted gastrectomy for gastric cancer and its clinical significance. World J Gastroenterol 2011; 17: 372-8. 